\title{
Brief report on ELCO and Its Plasticizing effects on PVC material
}

\author{
$\mathrm{Li} \mathrm{Ke}^{1, \mathrm{a}}$, Nie Xiao-an ${ }^{2, \mathrm{~b}}$ and Jiang Jianchun ${ }^{3, \mathrm{c}}$ \\ ${ }^{1}$ Institute of Chemical Industry of Forest Products, CAF, Nanjing, China \\ ${ }^{2}$ Institute of Chemical Industry of Forest Products, CAF, Nanjing, China \\ IInstitute of Chemical Industry of Forest Products, CAF, Nanjing, China \\ aliketaiping@163.com, ㅁniexiaoan@126.com, cbio-energy@163.com
}

Keywords: plasticize; epoxidation; ELCO; PVC; soybean oil.

\begin{abstract}
Choose soybean oil as raw material, through transesterification, epoxidation reaction, synthesized a new epoxy plasticizer - epoxy low calorie oils(ELCO), which molecular weight is somewhere in between ESO and EFAME, so it should be an excellent plasticizer product. Through the testing of some performance a conclusion about its application feasibility was given.
\end{abstract}

\section{Introduction}

Quality safety of PVC has become dominate problem which is concerned because a grate deal of plasticizers were applied to. An expert study concluded that environment-friendly plasticizers are the worldwide development trend and accord with sustainable development. One of which are epoxy oil-base plasticizers. In the commonly used epoxy oil-base plasticizer include epoxidized soybean oil $(E S O)^{[1]}$ and epoxy fatty acid methyl ester (EFAME) ${ }^{[2]}$ and occupies the majority. Although the ESO and EFAME has certain compatibility with polyvinyl chloride (PVC) and plasticy, and it's renewable and environmental protection. But several disadvantages tend to limit the use of them. Such as the compatibility of ESO with PVC is poorer, it usually used as adjuvant plasticizers, such as with DOP, and small addition quantity; Though EFAME has a better compatibility with PVC, and adding quantity can reach $40 \%$, to some degree it can alternative DOP produce non-toxic, environmental protection PVC product, and even can be used for food packaging, medical treatment, water supply pipeline, toys and other products, but EFAME molecular weight is too low, leading to the flash point is hard to rise, and limit the scope of application.

Low calorie oils and fats ${ }^{[3]}$ as a kind of relatively healthy oil in the food industry has been widespread attention, and have been thoroughly researched. Its molecular weight is slightly lower than that of soybean oil but higher than that of fatty acid methyl ester (FAME), so if use it as the raw material synthesize oil-base epoxy plasticizer, it will not only has excellent plasticity, but also have different features which ESO and EFAME don't have.

However the concept of low calorie oil (LCO) was barely known in the plastic process industry, it is almost no studies, so this article is to introduce the excellent varieties of plasticizer, and named it as epoxy low calorie oil (ELCO). In addition, choose soybean oil as raw materials synthesized a representativeness product - epoxy acetyl low calorie oil, and tested some important properties of plasticized PVC material. This will hopefully the ELCO can be given attention and industrial use, later we will do more research.

\section{Synthetic process}

Add soybean oil and glyceryl triacetate(mole ratio of soybean oil to glyceryl triacetate is $1: 1$ ) to a $500 \mathrm{~mL}$ three-necked round-bottomed with flask reflux condensing tube, adding the sodium methylate $\left(1 \mathrm{wt} \%\right.$ ) as catalyst, whizz until blend, heating up the mixture to $120^{\circ} \mathrm{C}$, and react $90 \mathrm{~min}$. Cooling and filtration, then wash it for 2 times with clean water for removing the sodium methylate, and vacuum distillation to $140{ }^{\circ} \mathrm{C}$ until no fractions, the residuum is LCO. 
Add $24 \mathrm{~g}$ of LCO to a $250 \mathrm{~mL}$ three-necked round-bottomed flask, at room temperature under magnetic stirring dripping a mixed mCPBA-methylene chloride solution slowly, and react 2-4 hour. Cooling and filtration, then alkali washing and wash it for 3 times with clean water till the $\mathrm{pH}$ value of water is equal to 7 , and dispose of solvent and water by distillation using a water ring vaccum pump (Nanjing, China) at $90^{\circ} \mathrm{C}$ under $-0.9 \mathrm{MPa}$. The residuum is ELCO. The reaction route is shown in Fig 1.
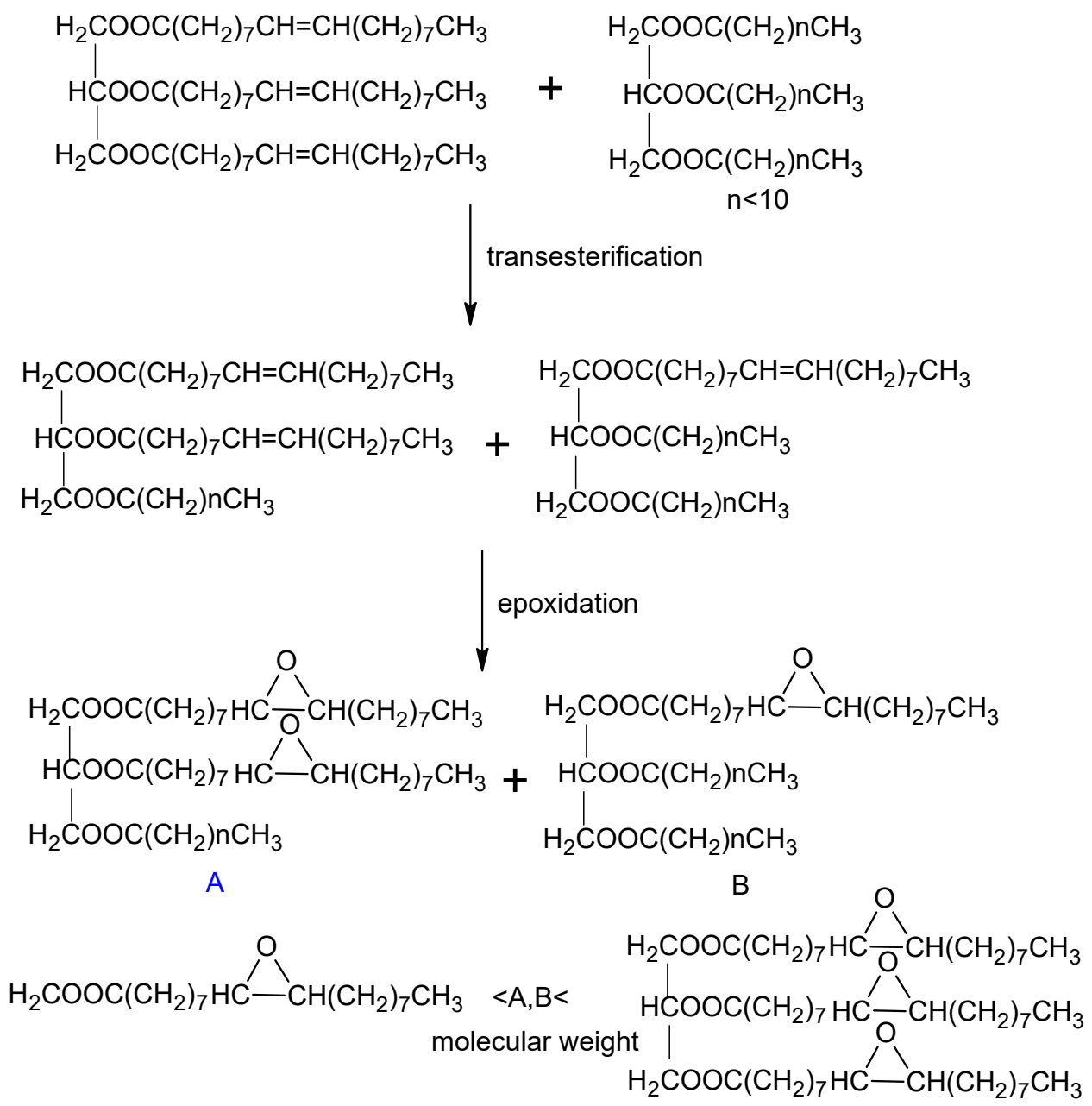

Fig.1 Synthetic route map

Fig 1 shows the structure of A (Double fatty acids type) and B (One fatty acid type) are similar to EFAME and ESO, and its molecular weight is intermediate between EFAME and ESO. So it should have good plasticity and new features.

\section{Structural characterization}

The analysis of molecular weight of LCO were performed by means of gel chromatography and shown in Fig 2, and the structure of synthesized ELCO was determined by IR (see Fig 3). 


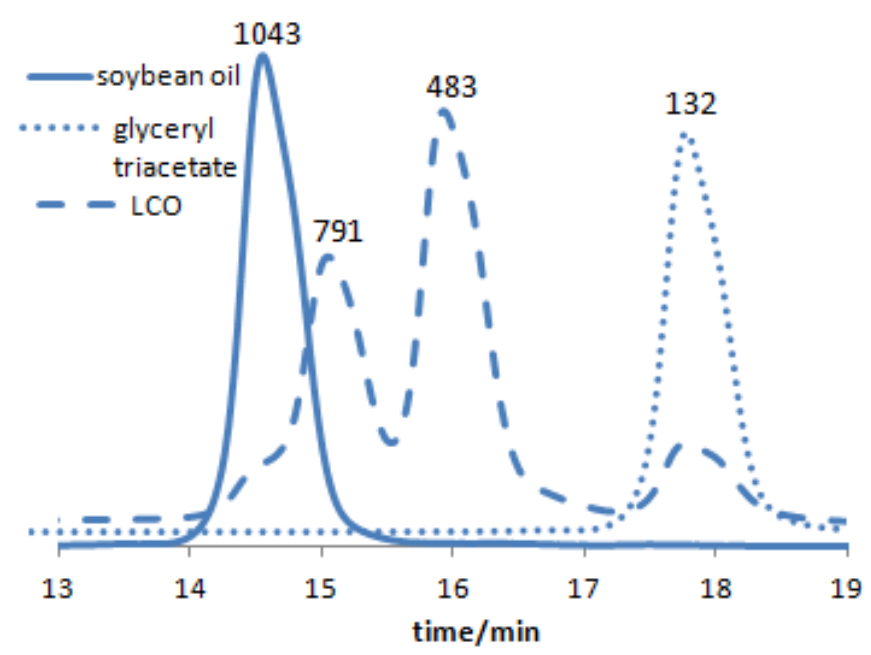

Fig. 2 Gel chromatogram of fresh and product

The GPC peak shifted according to molecular weight information of chemical compound. In Fig 2, full line and dashed line represented soybean oil and glyceryl triacetate, and the peak of LCO was between them, the peak of 791 is double fatty acids type, the peak of 483 is one fatty acid type, it's accord with the theory. so the synthetic technology is successful.

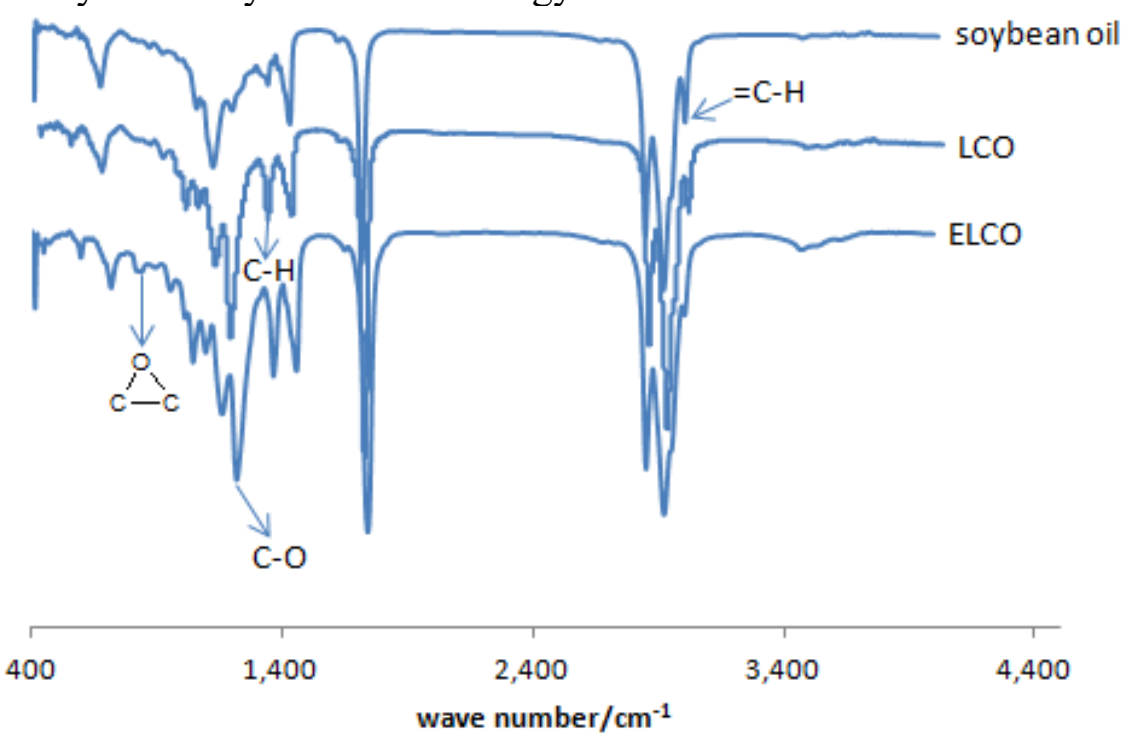

Fig.3 FTIR of oil, LCO and ELCO

Look at Fig.3, compared between soybean oil and LCO, in the spectrum of LCO, C-H groups deformation at $1360 \mathrm{~cm}^{-1}$, this is due to the increase of methyl groups after the carbon chain is shortened. C-O stretching at $1220 \mathrm{~cm}^{-1}$, this is the characteristic peak of acetate. This suggests that soybean oil has successfully synthesizing LCO; in the spectrum of ELCO, which indicated the existence of characteristic groups of epoxy bond $\left(814 \mathrm{~cm}^{-1}\right.$ and $\left.944 \mathrm{~cm}^{-1}\right)$, and the vanish of characteristic absorption peak of the $=\mathrm{C}-\mathrm{H}\left(2970 \mathrm{~cm}^{-1}\right)$. This shows that the product is really the ELCO.

\section{Performance test}

The glass transition temperature and elongation at break are the main indicators for plasticity. So in order to determine ELCO affects the PVC, we choose DMA to test all PVC films, including EFAME, ESO and ELCO. And the changes of loss tangent (tan $\delta$, glass transition temperature) are shown in Fig.4; And adopt Mechanics Performance Testing to all PVC films, including EFAME, ESO and ELCO, with the test outcome shown in Table 1. 


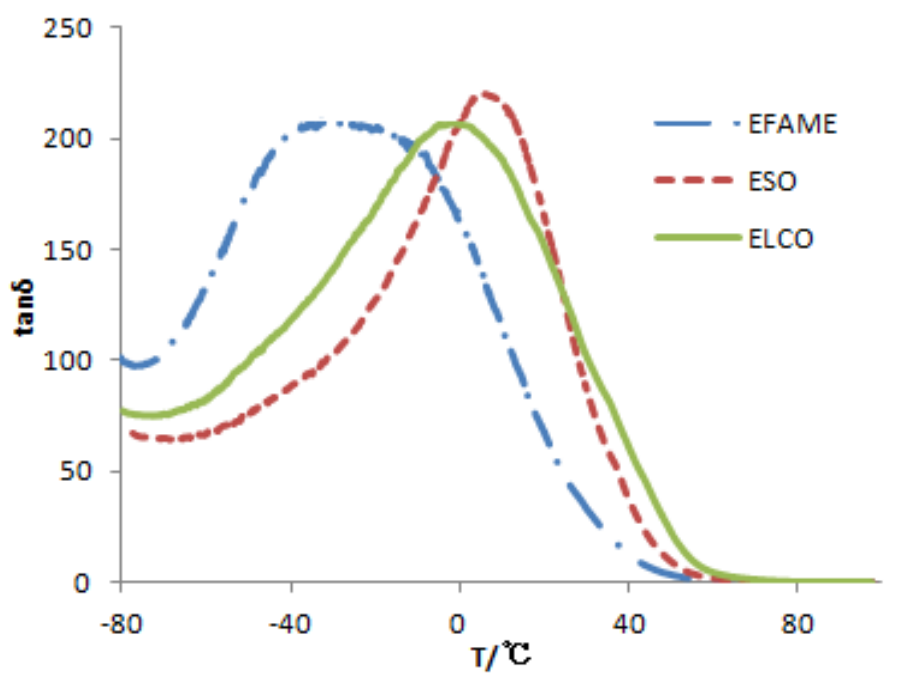

Fig.4 DMA $(\tan \delta)$ of PVC films

From Fig. 4 we can see that, the glass transition temperatures $(\tan \delta)$ of ELCO plasticizer PVC films $\left(-0.77^{\circ} \mathrm{C}\right)$ is intermediate between EFAME $\left(-29.94^{\circ} \mathrm{C}\right)$ and ESO $\left(6.13^{\circ} \mathrm{C}\right)$, which shows that its plasticizing effects on PVC is intermediate between EFAME and ESO; In addition, the height of the peaks of ELCO is similar to EFAME and lower than the ESO significantly, this suggesting that the macromolecule of ELCO plasticized PVC material is easier to relaxation then the ESO plasticized PVC, that is ELCO have better plasticizing performance than ESO.

Table 1: tensile test results

\begin{tabular}{ccccc}
\hline \multirow{2}{*}{ name } & ply & Max Force & $\begin{array}{c}\text { tensile } \\
\text { strength }\end{array}$ & elongation at break \\
\cline { 2 - 5 } & $\mathrm{mm}$ & $\mathrm{MPa}$ & $\mathrm{MPa}$ & $\%$ \\
\hline DOP & 0.24 & 25.53 & 26.22 & 360.64 \\
EFAME & 0.24 & 21.39 & 22.28 & 372.24 \\
ESO & 0.26 & 28.69 & 27.59 & 321.11 \\
ELCO & 0.26 & 28.60 & 27.88 & 370.56 \\
\hline
\end{tabular}

In Table 1, the ply, maximum force and tensile strength of ELCO plasticized film are similar to that of DOP plasticized film, and much bigger than that of EFAME and DOP; and the elongation at break is much bigger than that of ESO and EFAME. This also shows that ELCO has excellent plasticizing.

\section{Summary}

This study aims to introduce a new kind of epoxy oil-base plasticizer products - ELCO, and choose soybean oil and glyceryl triacetate synthesized a representativeness product - epoxy acetyl low calorie oil. The structure of product was identified by gel chromatography and IR, and tested glass transition temperature and elongation at break which are the main indicators for plasticity. The results showed that, the soybean oil can synthesis ELCO effectively, and it can reduce the glass transition temperature of PVC effectively, the PVC membrane has a larger elongation at break, so ELCO has excellent plasticizing. It's a more excellent epoxy plasticizer products, especially can be used as intermediate of ESO and EFAME, and make up the inadequacy of both.

\section{Acknowledgements}

This work was financially supported by the financial support from Key and Open Lab. of Biomass Energy and Material, Jiangsu Province, (Grant number: JSBEM-S-201704) and Natural Science Foundation of China (51761145014). 


\section{References}

[1] Buenoferrer C, Garrigós M C, Jiménez A, et al. Characterization and thermal stability of poly(vinyl chloride) plasticized with epoxidized soybean oil for food packaging[J]. Polymer Degradation \& Stability, 2010, 95(11):2207-2212.

[2] Cheng Z, Gong K, Wang Y, et al. Research Development on the Synthesis of Epoxy Fatty Acid Methyl Ester[J]. Plastics Additives, 2013.

[3] Akoh C C. New developments in low calorie fats and oils substitutes[J]. Journal of Food Lipids, 1996, 3(4):223-232. 